\title{
A Multiscale Communications System Based on Engineered Bacteria
}

\author{
Ozan Furkan Sezgen, Omer Altan, Ahmet Bilir, Merve Gorkem Durmaz, Nedim Haciosmanoglu, Berk Camli, Zeliha Cansu \\ Canbek Ozdil, Ali E. Pusane, Arda Deniz Yalcinkaya, Urartu Ozgur Safak Seker, Tuna Tugcu, and Sema Dumanli
}

The authors propose two different mechanisms to sense the output of the molecular communication system and transmit the information to an on-body reader. Each mechanism involves different genetically engineered bacteria and specific antenna designs. An experimental setup is provided to demonstrate each proposed concept. The results constitute a proof of concept to detect the in-body bacterial activity from the on-body reader.

\begin{abstract}
Although molecular communication systems have been shown to bear great potential for many useful in-body applications, they require the intervention, action, or input of an out-of-body actor. From an Internet of Bio-Nano Things perspective, a successful overall network aims to bring together the two links belonging to the in-body and out-of-body networks for end-to-end communications. For most applications, the uplink from the in-body sensor is more significant since it provides the multi-scalar connection required to relay the information sensed and carried by the molecular communication system to a macro-scale smart terminal. This article proposes two different mechanisms to sense the output of the molecular communication system and transmit the information to an on-body reader. Each mechanism involves different genetically engineered bacteria and specific antenna designs. An experimental setup is provided to demonstrate each proposed concept. The results constitute a proof of concept to detect the in-body bacterial activity from the on-body reader.
\end{abstract}

\section{INTRODUCTION}

Biology-inspired communication has drawn considerable attention in recent years due to the recent development in nanotechnology. Molecular communications (MC) at the nano-scale and body area networks (BANs) at the macro-scale have demonstrated high potential in communications research for health applications. In many applications, the missing link is the bridge between $M C$ and BAN, where the information gathered at the nano- or micro-scale is made accessible to the macro-scale BAN and its extensions.

Wireless sensing systems provide multi-scale communications and could be utilized to bridge this link and utilize advanced medical detection mechanisms to provide the best healthcare possible to patients in the field of diagnosis. This problem could also be viewed from an Internet of Bio-Nano Things (IoBNT) perspective [1], where in vivo sensors are utilized for real-time monitoring of patients' activity. The challenge here is to build a conventional wireless link between a biocompatible in-body sensing module and an on-body reader antenna. The sensing module is expected to be sensitive enough to accurately track the symptoms at the molecular scale. For this purpose, we utilize engineered bacteria to carry out the sensing tasks. Engineered bacteria can be programmed as whole cell biosensors (WCBs), and they can be used for a wide range of sensing/responding types of applications [2]. Such designs can be utilized in detecting many disease-related biomarkers, such as cancer-related, metabolic-diseases-related, and infectious-disease-related markers [3]. These input signals can be used to trigger many downstream processes within the cells such as drug secretion.

Although implantable sensors have high potential in real-time monitoring of patients' health, there are several drawbacks that hinder their widespread use. In addition to power requirements, the main problem is biocompatibility. Most in-body sensors require surgical removal to ensure that the device leaves no toxic traces behind. Also, the wireless link between the sensor and the on-body reader suffers from several degrading factors, such as high path loss and low antenna efficiencies. High path loss is a result of the high permittivity and conductivity of human tissues, and low implant/on-body antenna efficiencies are mainly caused by the small form factors needed for implantable and wearable devices and nearfield losses.

This article proposes two different sensing concepts, both of which utilize genetically modified bacteria to sense biological processes and specific chemical outputs produced by molecular communications. Proposed designs include a traceable signal generated by or reflected from an implantable device and an on-body reader antenna that can translate the molecular signal to a smart monitoring device via a conventional wireless link (Fig. 1).

Our first proposal utilizes genetically engineered fluorescent bacteria to produce a signal that will be transmitted to the on-body reader. The use of fluorescent proteins, a technique introduced to these bacterial strains in the 2000s, has led to the development of many applications, including biological sensors, tracking-visualization systems, and many other sophisticated biological devices [4, 5]. With the emergence of synthetic biology, control of MC as well as the control of fluorescent signals from bacteria can be achieved with high precision and programmability for many 
different molecules. Although engineered fluorescent bacteria have been used for in-body sensing applications, real-time in-body monitoring is quite a challenge, and there are only a few examples in the literature [6].

The proposed design is an active implant that requires a power source. Despite its high performance, it requires surgical removal after the monitoring period ends. To overcome these limitations, a second design is proposed using a passive and biodegradable device, as presented in Fig. 2. One frequently utilized approach to avoid the need for surgical removal is to utilize biodegradable sensors [7]. This work relies on the biodegradation of the sensor after its time of operation is complete. In order to realize a passive implant, backscatter-communications-based studies are also present [8], but these studies usually do not include biodegradability. Backscatter communications use a remote reader, which sends an electromagnetic wave toward a passive device and extracts the data collected by the passive device from the reflected electromagnetic wave. Reference [7] brings together biodegradation with backscatter communications but only presents simulation results [9]. One of the novel aspects of our approach over the state of the art is that rather than waiting for the sensor to biodegrade after sensing is complete, the proposed design measures the process/speed of biodegradation itself for the sensing task. In addition, to the best of our knowledge, this is the first time the utilization of genetically engineered bacteria for such a system is proposed. The bacteria is envisaged to be sensitive to a specific molecule of interest such as a molecule that is used as the messenger molecule in an MC scenario.

The rest of the article is organized as follows. We introduce the proposed methodology and discuss envisaged solutions and challenges. Then the experimental setup is demonstrated in detail, and the results are presented. Finally, we conclude the article.

\section{METHODOLOGY}

Both of the proposed mechanisms will utilize engineered Escherichia coli (E. coli). E. coli is a natural bacteria that has a simple genomic and metabolic structure as it can be easily grown and manipulated. Consequently, it is the most abundant model and working organism of molecular biology.

The proposed approaches tackle the challenges resulting from operating in an in-body environment, such as battery lifetime and biocompatibility. Details of each approach are provided in the following sections.

\section{ActIVE In-Body Sensing MeCHANISM}

The active sensing mechanism is envisaged to include a biofilm of engineered E. coli, which will produce fluorescent protein upon sensing the molecule of interest, and a transmitter that transmits an electromagnetic signal modulated according to the fluorescence of the biofilm through a photodetector, as shown in Fig. 2.

The transmitter will incorporate a photodiode (PD) to detect the fluorescence. The photodiode's transduction output will then be translated into an oscillating electrical signal whose frequency depends on the photodiode output. Oscillat-

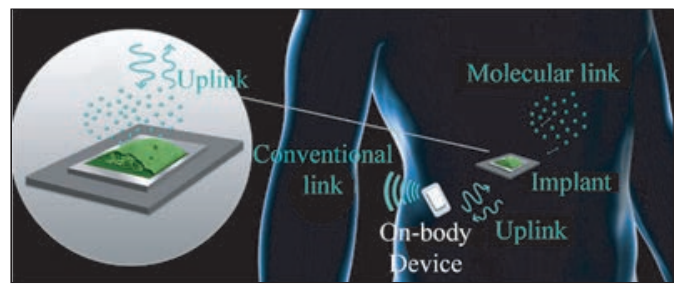

FIGURE 1. The conceptual representation of in-body sensors connecting the molecular link with BANs.

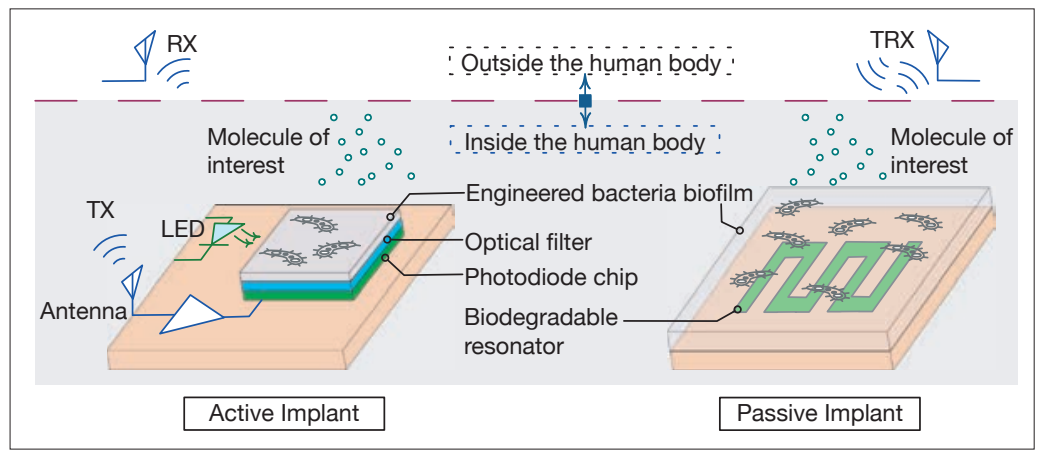

FIGURE 2. Schematic of proposed frameworks for molecular communication scenarios.

ing signals will be transmitted wirelessly with an implant antenna. Note that the photoexcitation of the fluorescent bacteria is envisaged to be done with incorporation of a light emitting diode (LED).

To realize the implant, all of the required components, including the photodiode, the power supply (SMD battery), the electronic components, the antenna, the light source (LED), and the bacterial biofilm, can be integrated on a biocompatible substrate (e.g., polyimide) to operate as a biosensing platform. The use of the battery is vital to provide power to the whole system during the monitoring period. This design requires surgical removal after the completion of the monitoring period. Such a platform can be applied for a variety of biosensing processes. While the main components of the sensing platform remain similar, the engineered bacteria can be altered to detect the presence of various molecules of interest.

In this work, for the case of active sensing, a harmless strain of genetically engineered E. coli is used to produce a measurable red fluorescent signal in the presence of the molecule of interest, Isopropyl $\beta$-D-1-thiogalactopyranoside (IPTG). By exciting and tracking the emitted signal coming from that red fluorescent protein, it is possible to detect the molecule of interest.

WCB sensors can be programmed to detect/ report many medically important biomarkers, which makes them excellent biological devices for early disease detection. Many fluorescent proteins or functional enzymes can be employed as reporters for WCB sensors [2]. In our case, fluorescent proteins are used, and they could be excited with simple LEDs. The optical signal generated due to red fluorescent protein excitation can be sensed and converted to an electrical signal via a photodiode, which performs signal transduction. Its operation principle relies on the interaction of a semiconductor junction with photons [10]. Incident photonic radiation at energy levels $(h v)$ greater than the band gap energy (Eg) of a semiconductor crystal generates electron-hole pairs (EHPs). These carriers give rise to a drift current 


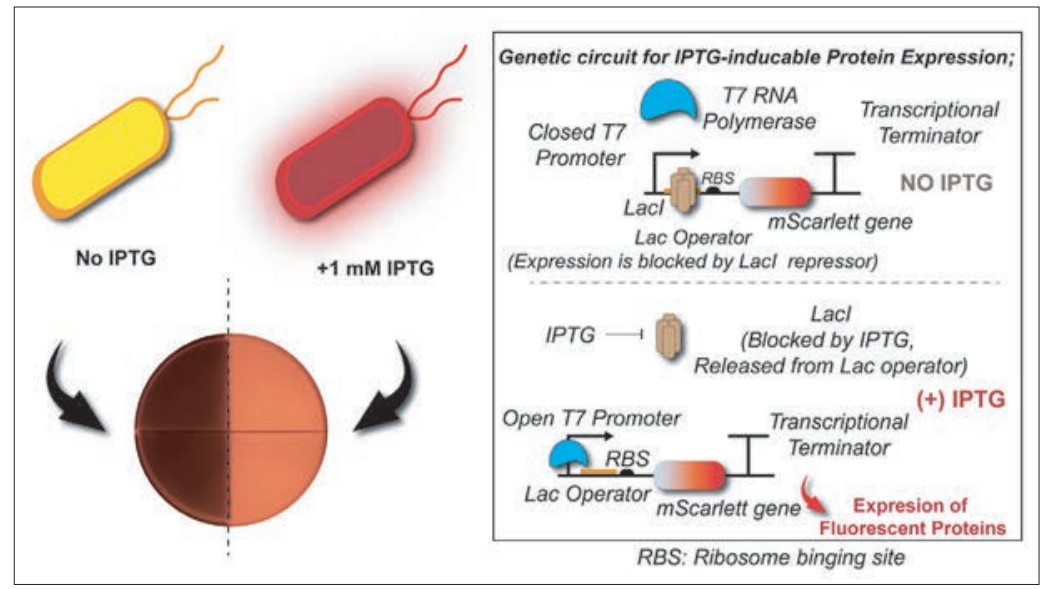

FIGURE 3. Genetically engineered, fluorescent E, coli strains and the genetic circuits for IPTG inducible fluorescent signal production. by the on-body transceiver. A biocompatible photoresist (SU-8) layer will be structured with lithographic definition as an etch mask on top of the layer to be protected from the biodegradation process. The whole sensor system is foreseen to be implemented on a biocompatible silk fibroin substrate. As the degradation evolves, the geometry of the resonator will be partially preserved, yielding a shift in the resonance frequency. A pair of on-body antennas is used to track this shift in transmission spectra. The wireless link established between these antennas and their interaction with the Mg implant will allow monitoring of the molecule of interest in-body in real time.

\section{EXPERIMENTAL SETUP AND RESULTS}

Initial experiments were conducted to realize proof of concept for both active and passive sensing mechanisms. In the active sensing platform, we present the feasibility of conversion of the molecular signal to an electrical one. In the case of passive sensing, we demonstrate the link between the biodegradation speed and bacterial activity. We also demonstrate tracking of biodegradation from outside a human body phantom wirelessly.

\section{Active In-Body Sensing SYSTem Design And MeasuREMENTS}

Utilization of Bacteria: The measured fluorescent signal is obtained from E. coli bacteria producing red fluorescent protein $\mathrm{mScarlet}$ [12] in the presence of IPTG molecules (Fig. 3). The easiest way to give additional functionalities to the bacteria in synthetic biology is by giving information for these functionalities with a DNA-carrying module, named plasmid DNA in the literature. Plasmids can be uptaken by the bacteria, and the information encoded in the plasmid DNA can be interpreted and produced by cellular machinery. For triggering the production of $\mathrm{mScarlet}$ with the molecule of interest IPTG, pET22b plasmid is used with some modifications, such as the final carrier having a complete genetic circuit, which is closed when there is no IPTG, and opened with the addition of IPTG by blocking the repression of lacl on the protein production module [13]. Cloning of the plasmid construct required for this experiment is done by Gibson Assembly and verified by next-generation sequencing. The overall genetic mechanism, which could be used for other molecules of interest, is illustrated in Fig. 3.

Bacterial strains are grown in a Luria Bertani (LB) medium supplemented with appropriate antibiotics. Overnight culture of bacterial strains is obtained by 16 hours of growth time in a $37^{\circ} \mathrm{C}$ incubator. Fresh cultures are grown by 1:100 dilution of the overnight culture in fresh media for 2-3 hours to reach the logarithmic growth phase.

Macroscale Sensing Terminal: The fluorescent signal intensity is measured via the complementary metal oxide semiconductor (CMOS) photodiode structure reported in [14]. The CMOS photodiode uses a total footprint area of $1.05 \mathrm{~mm} \times 1.0$ $\mathrm{mm}$. The design is fabricated along with a relevant $0.18 \mu \mathrm{m}$ CMOS process monolithically integrated with the electronics on the chip. The photodiode's anode-cathode pins are accessed through wirebond contacts, making photodiode characterization in isolation from circuitry blocks possible. 
The bacteria requires excitation in a certain wavelength window and emits light at a different wavelength. To be able to distinguish the fluorescent signal emitted by the bacteria from the excitation, an optical bandpass filter is employed. A printed circuit board (PCB) compatible with fabricated integrated circuit (IC) chips is designed and used for the characterization of on-chip photodiodes. For the characterization steps, a Keithley 4200 SCS semiconductor parameter analyzer's source measure units (SMUs) are employed. During the measurements with bacteria strains, the PCB is placed on top of the container holding $40 \mathrm{~mL}$ liquid samples. The photodiode's positioning is done so that the photodiode is directly above the samples, facing them without being immersed in them. A non-conducting cylinder shell structure, a geometric fit to the container rim, is mounted on the PCB to ensure mechanical stability during the measurements. Photoexcitation of bacteria for fluorescent signal observation is done with a green LED. It is positioned at the bottom level of the container in such a way that it does not directly face the photodiode. In addition, the optical bandpass filter is placed between the LED and the PCB. This is done in order to suppress the photodiode response to green light emitted by the LED so that the response to red fluorescent light can be observed without saturating the photodiode.

Setup components are placed within a dark chamber, where electrical connections are made through the conductive walls. Exploiting this, measurements are performed in isolation from all other light sources, while LED control and photodiode characterization are done in a separate room. The experimental setup is schematized in Fig. 4.

Experimental Results: The short circuit photodiode current (Isc) of the photodiode is traced as the sensor output. According to the theory described above, the decrease in Isc corresponds to increased levels of light intensity. The first experimental results are presented in Fig. 4. Five measurements in each case are performed. Error bars show the standard deviation of the measurements. Statistical analysis is made by non-parametric t-test. In the presence of light from the green LED, photodiode responses to different cases vary. In the first 2 hours, the photodiode response to the reference and IPTG induced samples is almost the same, and Isc currents are slightly over $0.5 \mathrm{nA}$ for both. This is expected, since the engineered bacteria increases its signal in the later phases of its growth by opening fluorescent protein production machinery when the IPTG is presented in our case. The IPTG effect on the sample is increased after four hours, and it leads to the difference observed in Fig. 4 (right) between the Isc of two samples. Isc caused by the IPTG-induced sample exceeds $1 \mathrm{nA}$, whereas the reference sample remains below this threshold. The trend prevails until the end of the monitoring period.

\section{PaSSIVE BIODEgRadable IN-BodY SeNSOR SYSTeM DESIGN AND MEASUREMENTS}

Utilization of Bacteria: Tracking the $\mathrm{Mg}$ implant's degradation speed is planned to be used as the sensing principle of bacterial activity [15]. Here, the correlation between the bacterial
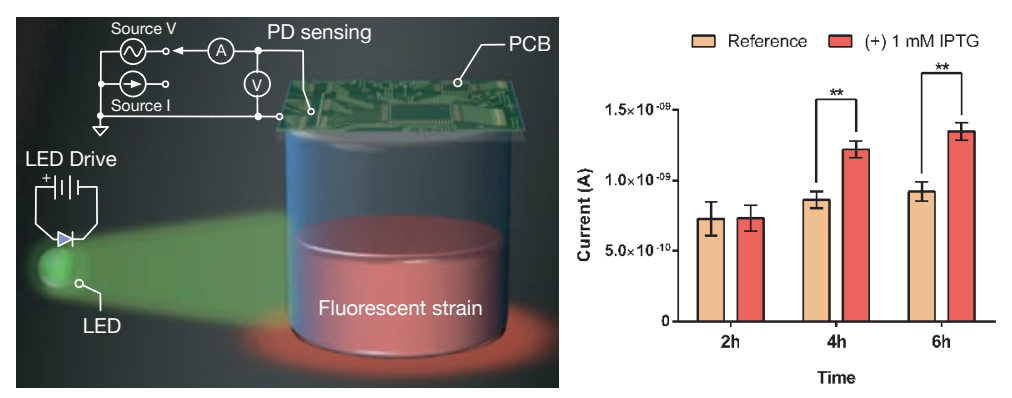

FIGURE 4. Schematic showing: left: measurement setup (constant current LED drive and SMU based PD sensing) for active in-body sensing system; right; first measurement results.

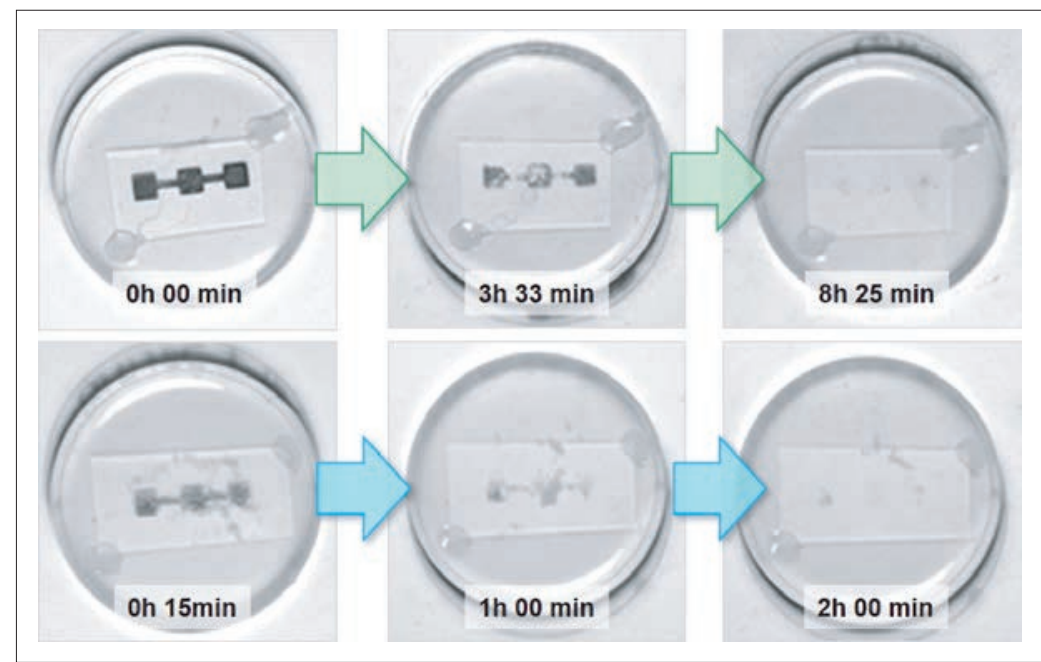

FIGURE 5. The effect of bacterial activity on biodegradation speed:top: FBS; bottom: FBS with E. coli.

activity of $\mathrm{E}$. coli and the degradation is demonstrated in fetal bovine serum (FBS).

To observe the effect of bacteria on $\mathrm{Mg}$ degradation clearly, thin profile $\mathrm{Mg}$ structures were used, reducing implant lifetime, permitting easier tracking of smaller changes, and retarding the formation of the $\mathrm{Mg}(\mathrm{OH}) 2$ layer on the implant, which interferes with the degradation process. The Mg implants, as seen in Fig. 5, are produced via direct current (DC) magnetron sputtering on a glass substrate, which makes degradation visible to the naked eye. The implant is designed as a bridge-like structure, with a combination of thick and thin parts, to test the functionality of sensor shape and the concept of a gradual degradation approach. The implants were sterilized before interacting with FBS and bacteria. Among different sterilization methods, such as steam autoclave, dry heat, formaldehyde, and ultraviolet (UV) light sterilizations, UV light sterilization is chosen here due to its minimal impact on the structural level. Mg implants fixed into Petri dishes are kept under germicidal UV light for 30 minutes.

The biodegradation rate of the designed $\mathrm{Mg}$ implants in sterile FBS and E. coli added FBS (0.1:10) are compared, as can be observed in Fig. 5. The complete degradation process takes approximately four times less time in FBS with E. coli compared to sterile FBS. Results demonstrate that the presence of E. coli accelerates the degradation rate of the implant. Initial rupture occurs in the thin connective layers, while the rest of the implant degrades in a nonhomogeneous manner 
Note that the high permit-

tivity and conductivity of

FBS prove sensing to be

difficult. The novelty in our

system not only lies in the

way we use biodegradation

for bacterial activity sensing,

but also in promising in-body

sensing with the utilization

of high-quality factor resona-

tors and improved on-body

antenna design.

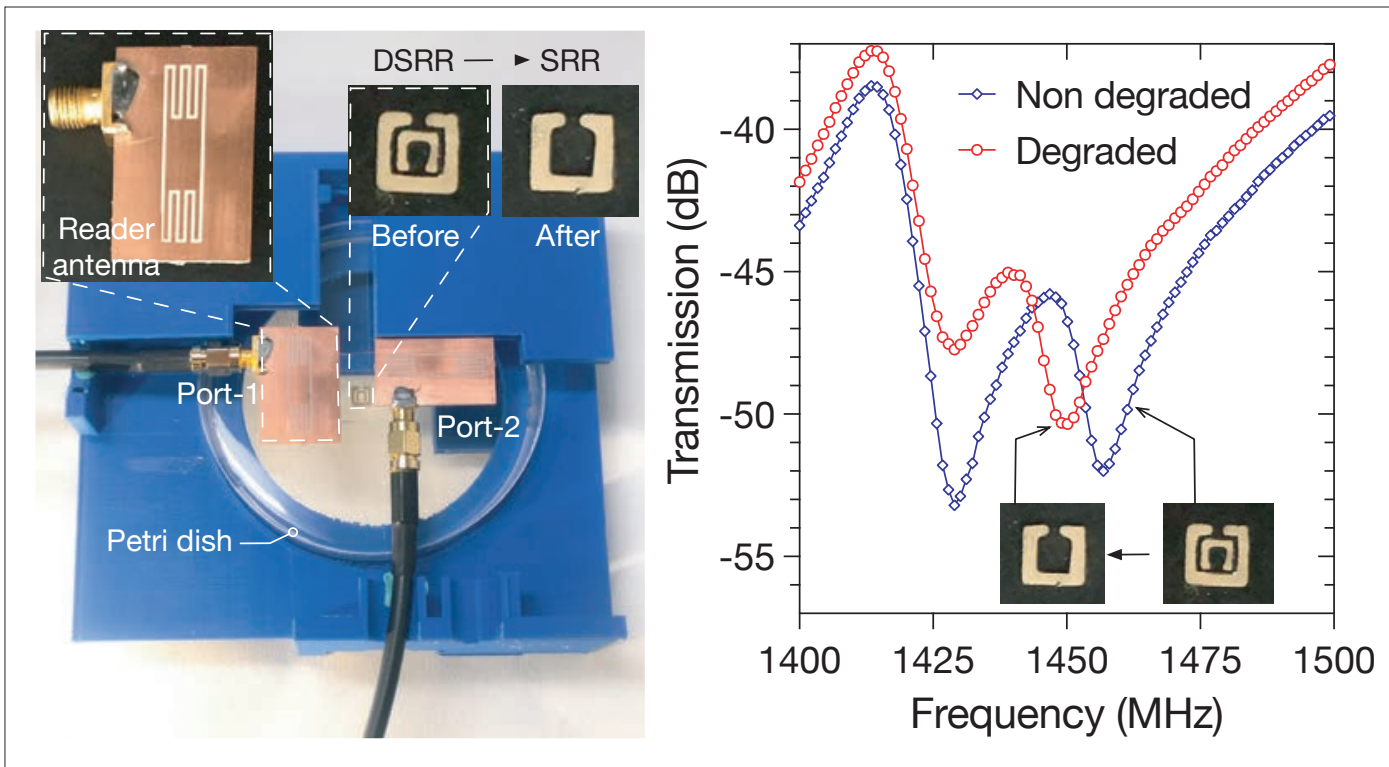

FIGURE 6. Left: schematic showing measurement setup for passive in-body sensing system including reader antennas, resonators at two different biodegradation phases submerged in FBS measured in Petri dishes; right: initial experimental results demonstrating the change in transmission $|s 21|$ in $\mathrm{dB}_{\text {." }}$

at a slower rate. As the bridges degrade in time, the magnesium implant loses its structural integrity right before complete dissolution.

Macroscale Sensing Terminal: Building onto these results, an implantable double split-ring resonator (DSRR) and an on-body meandered slot loop antenna are designed. A pair of these on-body reader antennas is located outside the FBS (representing the human body phantom). The wireless link between them and its interaction with the implantable resonator changes as degradation occurs. Note that the high permittivity and conductivity of FBS prove sensing to be difficult. The novelty in our system lies not only in the way we use biodegradation for bacterial activity sensing, but also in promising in-body sensing with the utilization of high-quality factor resonators and improved on-body antenna design.

Experimental Results: The operating frequency of the resonator, which should change as it degrades, is tracked, as seen in Fig. 6. The experimental setup includes the aforementioned on-body antennas, a Petri dish where the implantable resonator is merged in FBS, the resonator, and a vector network analyzer (VNA). To represent different phases of biodegradation, two split ring resonators (SRRs) are prototyped on glass with conductive ink via PCB Printer. The first one, with two rings, represents the DSRR before degradation, while the second has only the outer ring, representing the SRR version. These two models represent two separate phases of the resonator. The reader antenna is etched on RO3210 high-frequency laminate using PCB Prototype Technology.

SRR structures representing two phases of degradation are placed in FBS with a relative permittivity $\left(\varepsilon_{r}\right)$ of 66 and conductivity of $1.76 \mathrm{~S} / \mathrm{m}$. Figure 6 presents the change in the transmission behavior between the reader antennas with degradation. The time elapsed between these two phases can be mapped to bacterial activity, as proved from our Mg-Bacterial activity experiments.

\section{CONCLUSION}

This article presents two novel approaches to in-body sensing. The two mechanisms proposed to detect the molecule of interest via $\mathrm{E}$. coli can easily be integrated at the sink of a molecular communication system and can be used to link this end of the nanonetwork to BANs. One of the proposed methods employs active circuit components, resulting in the need for a battery, while the second one relies on passive components and is biodegradable. The experiments conducted for this study yielded encouraging results for both sensors. The active sensor was able to detect the molecule of interest, a crucial piece of information to be transmitted to the on-body reader. For the passive sensor, we were able to link bacterial activity to the transmission behavior of on-body reader antennas. Both approaches are thus shown to have the potential to bridge the missing information link between molecular nanonetworks and body area networks based on engineered bacteria.

\section{ACKNOWLEDGMENTS}

This work has been supported partially by the Turkish Directorate of Strategy and Budget under the TAM project number DPT2007K120610 and Bogazici University Research Fund under project number 14543. We thank Oguz Kaan Erden for his major contribution to the project.

\section{REFERENCES}

[1] I. F. Akyildiz et al., "The internet of Bio-Nano things," IEEE Communications Mag., vol. 53, no. 3, Mar. 2015, pp. 32-40. DOI: 10.1109/MCOM.2015.7060516.

[2] B. Saltepe et al. "Genetic Circuits Combined with Machine Learning Provides Fast Responding Living Sensors," Biosensors and Bioelectronics, vol. 178, Apr. 2021, p. 113028. DOI: 10.1016/j.bios.2021.113028.

[3] B. Saltepe et al., "Cellular Biosensors with Engineered Genetic Circuits," ACS Sensors, vol. 3, no. 1, Jan. 2018, pp. 13-26. DOI: 10.1021/acssensors.7b00728.

[4] S. Slomovic, K. Pardee, and J. J. Collins, "Synthetic Biology Devices for In Vitro and In Vivo Diagnostics," Proc. Nat'l. Acad. Sci. USA, vol. 112, no. 47, Nov. 2015, p. 14,429. DOl: 10.1073/pnas.1508521112. 
[5] O. V. Stepanenko et al., "Fluorescent Proteins as Biomarkers and Biosensors: Throwing Color Lights on Molecular and Cellular Processes," Curr Protein Pept. Sci., vol. 9, no. 4, Aug. 2008, pp. 338-69. DOI: 10.2174/138920308785132668.

[6] M. Mimee et al., "An Ingestible Bacterial-Electronic System to Monitor Gastrointestinal Health," Science, vol. 360, no. 6391, May 2018, p. 915. DOI: 10.1126/science.aas9315.

[7] C. M. Boutry et al., "A Stretchable and Biodegradable Strain and Pressure Sensor for Orthopaedic Application," Nature Electronics, vol. 1, no. 5, May 2018, pp. 314-21. DOI: 10.1038/s41928-018-0071-7.

[8] A. Khaleghi, A. Hasanvand, and I. Balasingham, "Radio Frequency Backscatter Communication for High Data Rate Deep Implants," IEEE Trans. Microwave Theory and Techniques, vol. 67, no. 3, Mar. 2019, pp. 1093-1106. DOI: 10.1109/TMTT.2018.2886844.

[9] J. M. Rigelsford et al., "A Passive Biodegradable Implant for Subcutaneous Soft-Tissue Trauma Monitoring," IEEE J. Biomedical and Health Informatics, vol. 19, no. 3, May 2015, pp. 901-09. DOI: 10.1109/JBHI.2015.2417754.

[10] S. M. Sze, Physics of Semiconductor Devices, 2nd ed., Wiley, 1981

[11] J. Y. Lock et al., "Degradation and Antibacterial Properties of Magnesium Alloys in Artificial Urine for Potential Resorbable Ureteral Stent Applications," J. Biomedical Materials Research Part A, vol. 102, no. 3, Mar. 2014, pp. 781-92. DOI: $10.1002 / j b m . a .34741$.

[12] D. S. Bindels et al., "mScarlet: A Bright Monomeric Red Fluorescent Protein for Cellular Imaging," Nature Methods, vol. 14, no. 1, Jan. 2017, pp. 53-56. DOI: 10.1038/nmeth.4074.

[13] J. W. Dubendorf and F. W. Studier, "Controlling Basal Expression in an Inducible T7 Expression System by Blocking the Target T7 Promoter with LAC Repressor," J. Molecular Biology, vol. 219, no. 1, May 1991, pp. 45-59. DOI: 10.1016/0022-2836(91)90856-2.

[14] B. Camli, B. Sarioglu, and A. D. Yalcinkaya, "Photodiodes for Monolithic CMOS Circuit Applications," IEEE J. Selected Topics in Quantum Electronics, vol. 20, no. 6, Dec. 2014, pp. 336-43. DOI: 10.1109/JSTQE.2014.2333236.

[15] K. Ararat et al., "A Biodegradable Implant Antenna Detecting Post-Surgical Infection," 2020 14th Euro. Conf. Antennas and Propagation, Mar. 2020, pp. 1-4. DOI: 10.23919/ EuCAP48036.2020.9135877.

\section{BIOGRPPHIES}

OZAN FURKAN SEZGEN received his B.Sc. (Hons.) in electrical and electronics engineering from Bilgi University, Istanbul, Turkey, in 2018. He was a student assistant in his Bachelor's last year. He is currently an M.Sc. student in the Electronics Department at Bogazici University, Istanbul, Turkey, and is a member of the Antennas \& Propagation Research Laboratory (BOUNtenna).

OMER ALTAN is currently studying electrical and electronics engineering and mathematics at Bogazici University. He is a member of BOUNtenna. He received the Vali Celalettin Tufekci Merit-Based Scholarship, Huseyin Akif Terzioglu Foundation (2016-2020).

AHMET BILIR is currently studying electrical and electronics engineering and mathematics at Bogazici University. He is a member of BOUNtenna.

NEDIM HACIOSMANOGLU received his B.Sc. degree in molecular biology and genetics from Istanbul Technical University in 2016. He received his M.Sc. degree from the Materials Science and Nanotechnology program at Bilkent University in 2019. He is currently a Ph.D. student at the Synthetic Biosystems Laboratory of Bilkent University's National Nanotechnology Research Center led by Dr. Urartu Ozgur Safak Seker. His research topics include engineered probiotics, genetic circuits, and de novo design of engineered biological systems.

Merve Gorkem DuRMaZ received her B.Sc. degree in molecular biology and genetics in 2019 from Bogazici University. She has been a researcher in the Nanonetworking Research Group (NRG) at Bogazici University, Computer Engineering Department since 2018. She is currently an M.Sc. student at Bogazici University, Computer Engineering Department, and her research interests include molecular communications, microfluidics, and bioinformatics.

Berk CAMLI received his B.S. (Hons.), M.S., and Ph.D. degrees from the Department of Electrical and Electronics Engineering at Bogazici University in 2010, 2013, and 2020, respectively. He received the Turkish Prime Ministry Scholarship (2005-2010) and Is Bankasi Golden Youth Award in 2005. His research interests include photonics, metamaterial-based sensors, and microsystem design.

Zeliha CANSU CANBeK OZDIL received her B.Sc. degree in chemistry from Middle East Technical University and her M.Sc. degree from the University of Regensburg in the field of complex condensed materials and soft matter. She received her Ph.D. degree in theoretical chemistry from the University of Versailles-St-Quentin. She is currently working as a postdoctoral fellow at Bogazici University in the field of nanonetworking. Her specific area of research includes production/characterization of functional nanomaterials and development of microfluidic lab-on-chip (LOC) platforms.

ARDA DENIZ YALCINKAYA received his B.Sc. degree from Istanbul Technical University, Electronics and Telecommunication Engineering Department, and his M.Sc. and Ph.D. degrees from Technical University of Denmark, Department of Micro and Nano Technology, Lyngby, in 1997, 1999, and 2003, respectively. Between 2003 and 2006, he was a research associate at Koc University, Istanbul, and a consultant to Microvision Inc., Seattle, Washington. He has been a faculty member in the Department of Electrical and Electronics Engineering, Bogazici University since 2006. He is the recipient of Bogazici University Foundation's Distinguished Young Scientist Award, METU Mustafa Parlar Foundation Young Investigator Award, Turkish Academy of Sciences (TUBA) Young Scientists Award, and Elginkan Award. $\mathrm{He}$ is the co-founder of GlakoLens AS. His research interests include photonics, microsystems, metamaterials, and integrated circuits.

Ali EMre PusAne received his B.Sc. and M.Sc. degrees in electronics and communications engineering from Istanbul Technical University in 1999 and 2002, respectively, and his M.Sc. degree in electrical engineering, M.Sc. degree in applied mathematics, and Ph.D. degree in electrical engineering from the University of Notre Dame, Indiana, in 2004, 2006, and 2008, respectively. He was a visiting assistant professor with the Department of Electrical Engineering, University of Notre Dame during 2008-2009. He then joined the Department of Electrical and Electronics Engineering, Bogazici University. His research interests include wireless communications, molecular communications, information theory, and coding theory.

URARTU OzGUR SAFAK SEKER received his Ph.D. in molecular biology and genetics from Istanbul Technical University in 2009. He carried out his experimental studies at the University of Washington, Seattle between 2004-2008 in the Materials Science and Engineering Department. In March 2010 he became a postdoctoral research fellow in the Applied Physics Department at Nanyang Technological University, Singapore, and moved to MIT as a postdoctoral researcher, where he was affiliated with the Synthetic Biology Research Center, RLE and Biological Engineering. Since May 2014 he has been a professor at Bilkent University Institute of Materials Science and Nanotechnology Life Sciences Section. His research covers synthetic biology and its biomedical applications. He is a recipient of Distinguished Young Scientist awards from Turkish Academy of Sciences, Turkish Institute of Health, Science Academy, METU Parlar Foundation, and FABED.

TUNA TUGCU received his B.Sc. and Ph.D. degrees in computer engineering from Bogazici University in 1993 and 2001, respectively, and his M.Sc. degree in computer and information science from the New Jersey Institute of Technology, Newark, in 1994. He was previously a postdoctoral fellow and a visiting professor at Georgia Institute of Technology. He is currently a professor in the Department of Computer Engineering, Bogazici University. He is a faculty member of the Computer Networks Research Laboratory (NETLAB) and co-founded the Nanonetworking Research Group (NRG). His research interests include nanonetworking, molecular communications, wireless networks, and IoT. He has served with North Atlantic Treaty Organization science and technology groups and IEEE standards groups. He is an Associate Editor of IEEE Transactions on Molecular Biology and the Multi-Scale Communications Journal.

SEMA DUMANLI received her B.Sc. degree in electrical and electronics engineering from Orta Dogu Teknik Universitesi, Ankara, Turkey, in 2006, and her Ph.D. degree from the University of Bristol, United Kingdom, in 2010. She was with Toshiba Research Europe, Bristol, as a research engineer and a senior research engineer from 2010 to 2017. She is currently an assistant professor at Bogazici University. She is the founder of BOUNtenna. Her current research interests include antenna design for body area networks, implantable and wearable devices, eHealth, and molecular communications. 